\title{
Impulse für die Versorgungsforschung: Was folgt auf die DGPPN S3-Leitlinie zu psychosozialen Therapien?
}

\author{
Stimulating Mental Health Services Research: What are the Implications of the \\ New DGPPN S3-Guideline for Psycho-Social Therapies?
}

Autoren

Institute

\author{
Anke Bramesfeld ${ }^{1,2}$, Ingo Schäfer ${ }^{3}$, Katarina Stengler ${ }^{4}$, Georg Schomerus ${ }^{5}$ \\ 1 Institut für Epidemiologie, Sozialmedizin und Gesundheitssystemforschung, Medizinische Hochschule Hannover \\ AQUA-Institut für angewandte Qualitätsförderung und Forschung im Gesundheitswesen GmbH, Göttingen \\ 3 Klinik für Psychiatrie und Psychotherapie, Universitätsklinikum Hamburg-Eppendorf \\ Klinik und Poliklinik für Psychiatrie und Psychotherapie, Universitätsklinikum Leipzig, AöR \\ 5 Klinik und Poliklinik für Psychiatrie und Psychotherapie der Universitätsmedizin Greifswald am HELIOS Hanseklinikum \\ Strahlsund
}

\section{Bibliografie \\ DOI http://dx.doi.org/ \\ 10.1055/s-0033-1359949 \\ Psychiat Prax 2014; 41: 65-67 \\ (c) Georg Thieme Verlag KG \\ Stuttgart · New York \\ ISSN 0303-4259}

Korrespondenzadresse

Priv.-Doz. Dr. Georg Schomerus Klinik und Poliklinik für

Psychiatrie und Psychotherapie der Universitätsmedizin

Greifswald am HELIOS

Hanseklinikum Stralsund

Rostocker Chaussee 70

18437 Stralsund

georg.schomerus@uni-

greifswald.de
Es gibt ein breites und vielfältiges Angebot an psychosozialen Therapien in Deutschland. Die Inanspruchnahme dieser Therapien scheint sich aber weniger an wissenschaftlicher Evidenz als vielmehr an regionalen Gegebenheiten zu orientieren. Eine exemplarische Studie der Versorgungslage in 4 unterschiedlichen deutschen Regionen zeigte beispielsweise, dass es auch in strukturschwachen Regionen psychosoziale Angebote gibt, die Nutzung der Angebote aber starken regionalen Schwankungen unterliegt [1]. Psychosoziale Therapien sind in erster Linie eine Domäne der ambulanten Versorgung. Angesichts der fortschreitenden, durch die Einführung des neuen Entgeltsystems weiter forcierten Verkürzung der stationären Aufenthaltsdauern ist zu erwarten, dass die Bedeutung der ambulanten Versorgung und damit auch der psychosozialen Therapien weiter steigen wird. Dies gilt besonders für schwer und chronisch psychisch kranke Menschen. Für diese Klientengruppe entscheidet sich vor allem in der ambulanten Versorgung, inwieweit relevante Outcomes wie zum Beispiel Beschäftigung, selbstständiges Wohnen oder allgemein eine hohe Lebensqualität erreicht werdenRecovery findet nicht in der Klinik, sondern in der Gemeinde statt [2]. Psychosoziale Therapien spielen dabei eine zentrale Rolle, denn sie setzen da an, wo sich eine Erkrankung im Alltagsleben manifestiert.

Die neue S3-Leitlinie zu psychosozialen Therapien [3] bietet erstmals eine umfassende Würdigung der Evidenz für die Wirksamkeit psychosozialer Interventionen. Als dritte Säule therapeutischer Interventionen in der Psychiatrie neben den somatischen und den psychotherapeutischen Behandlungsangeboten erfahren psychosoziale Therapien damit nicht nur eine evidenzbasierte wissenschaftliche Aufwertung, sondern es besteht die Hoffnung einer höheren Akzeptanz dieser Verfahren in der psychiatrisch-psychotherapeutischen Community. In erster Linie bietet die neue
Leitlinie aber eine große Chance, die Versorgung schwer psychisch kranker Menschen mit psychosozialen Therapien zu verbessern. Sie bietet die Möglichkeit, durch einen Vergleich der aktuellen Versorgungslage mit den Leitlinienempfehlungen Stärken und Schwächen der aktuellen Versorgung abzubilden und die Richtung für zukünftige, evidenzbasierte Entwicklungen in der Versorgung vorzugeben. In einem Editorial in der Psychiatrischen Praxis haben Thomas Becker und Steffi Riedel-Heller, die maßgeblich an der Entstehung der Leitlinie beteiligt waren, die Frage aufgeworfen, wie die Versorgungsforschung die Implementierung der Leitlinie begleiten und unterstützen kann [4]. In diesem Beitrag wollen wir diese Frage aufgreifen. Wir möchten Überlegungen vorstellen, welche konkreten Fragestellungen sich für die Versorgungsforschung prioritär ergeben, um die Implementierung der Leitlinie zu unterstützen.

\section{Besonderheiten der Versorgung mit psychosozialen Therapien \\ $\nabla$}

Die in den Leitlinien gewürdigten, sehr heterogenen Therapien und Strukturen weisen mehrere Besonderheiten auf, die eine besondere Herausforderung für eine wissenschaftliche Bestandsaufnahme darstellen. Die Implementierung dieser Leitlinie unterscheidet sich dadurch erheblich von der Implementierung anderer Leitlinien. Das Spektrum der Leitlinie ist breit: Es reicht von den Grundlagen der therapeutischen Beziehung über Systeminterventionen wie Case Management, $\mathrm{Ar}$ beitsrehabilitation und Wohnangebote bis zu Einzelmaßnahmen wie Psychoedukation, Peer-topeer-Beratung, Selbsthilfe, Ergotherapie und künstlerische Therapien. Damit fokussiert die Leitlinie nicht nur auf diagnoseübergreifendes Denken und Handeln, sondern bezieht sich im besten Sinne auf multiprofessionelles, teamba- 
siertes Arbeiten im psychiatrisch-psychotherapeutischen Kontext. Jenseits einer traditionell ärztlich betonten Sichtweise werden hier auf evidenzbasierter Grundlage alle in der Psychiatrie und Psychotherapie tätigen Berufsgruppen und darüber hinaus auch Angehörige bzw. Peers angesprochen und ihre Rolle in der psychosozialen Versorgung adressiert. Indem verschiedene Akteure des sozialen Umfelds einbezogen werden, sind die Grenzen zwischen medizinischem Versorgungssystem und gesellschaftlichem Kontext fließend.

\section{Sektorenübergreifende Versorgung}

Viele Inhalte der Leitlinie beziehen sich auf Bereiche außerhalb der stationären Psychiatrie, aber auch außerhalb der krankenkassenfinanzierten ambulanten Psychiatrie. Der Grund dafür ist die Sektorisierung der psychosozialen Versorgung, bei der eine Vielzahl von gemeindepsychiatrischen Angeboten nicht über die Krankenkassen oder die Rentenversicherung, sondern kommunal oder länderspezifisch (im Bereich des SGB XII) geregelt ist. Eine angemessene Betrachtung der Versorgungsrealität muss also kostenträger- und anbieter- bzw. sektorenübergreifend erfolgen [5].

Dabei machen die Kostenträgerstruktur und die Anbietervielfalt eine Vernetzung und enge Abstimmung auf lokaler Ebene notwendig, die entscheidend für die Qualität der Versorgung ist. Fachärzte, medizinische und psychosoziale Einrichtungen, Betreuer, Vereine und nicht zuletzt die Betroffenen selbst können Knotenpunkte in solchen lokalen Netzwerken sein und damit die Art und Qualität der Versorgung vor Ort maßgeblich bestimmen. Das bedeutet, dass die Qualität der psychosozialen Versorgung auf der regionalen Ebene entsteht und maßgeblich von den lokalen Strukturen und den Akteuren vor Ort abhängig ist [1]. Eine Untersuchung der psychosozialen Versorgung muss daher die Region als Erbringer bestimmter Versorgungsleistungen in den Fokus nehmen.

\section{Starker Einfluss therapieferner Faktoren}

Umgekehrt bedeutet dies für die Implementierung der Leitlinie, dass die Vielfalt der Anbieter und Kostenträger dazu führt, dass Veränderungen auf ganz unterschiedlichen ordnungspolitischen Ebenen direkten Einfluss auf die Versorgung haben. Beispielsweise können Veränderungen der Verfügbarkeit von „Ein-EuroJobs“, der kommunalen Finanzierung von Hilfsangeboten, der Kostenübernahme von ambulanten Krankenkassenleistungen und die Bewilligungspraxis von Rehabilitationsmaßnahmen jeweils unabhängig voneinander Einfluss auf die psychosoziale Versorgung der Betroffenen haben. Im Vergleich zur Implementierung von Leitlinien in anderen Themenfeldern (z.B. auf dem Gebiet der Pharmakotherapie) erschwert die Vielfalt der Anbieter, Strukturen und Einflussgrößen die Dissemination und Implementierung der Leitlinie zu psychosozialen Therapien. Dies trifft auch für das Erheben relevanter Daten im Rahmen einer wissenschaftlichen Evaluation zu.

\section{Was steht drauf, was ist drin?}

Bei einer Untersuchung des Umsetzungsgrads einer Leitlinie müssen die Prozesse betrachtet werden, die tatsächlich bei der Behandlung von Klienten ablaufen. Es greift dabei zu kurz, diese Prozesse nur als Inanspruchnahme von Leistungen definierter Anbieter darzustellen. Psychosoziale Hilfen und Interventionen werden teilweise parallel von verschiedenen Anbietern unter verschiedenen Bezeichnungen angeboten, verfolgen inhaltlich aber dasselbe Ziel. So kann Alltagstraining als Leistung der ge- setzlichen Krankenkasse sowohl im Rahmen von Ergotherapie, Soziotherapie oder ambulanter psychiatrischer Krankenpflege angeboten werden; außerhalb des Bereichs der GKV kann Alltagstraining aber auch im Rahmen von betreutem Wohnen oder durch den sozialpsychiatrischen Dienst erfolgen. Unter dem Label „psychiatrische Krankenpflege“ können sich die verschiedensten Inhalte von der alleinigen Kontrolle der Medikamenteneinnahme bis hin zu intensivem Hometraining verbergen. Untersuchungen zur Implementierung der Leitlinie sollten daher von der Klientensicht ausgehen. Es interessiert vor allem, welche Interventionen und dazugehörigen Prozesse einen Klienten tatsächlich erreichen und erst in nachgeordneter Linie, wer die Leistung erbringt. Ob die Versorgungsleistungen für einen individuellen Klienten tatsächlich angemessen sind, kann im Einzelfall nur schwer entschieden werden. Die Angemessenheit einer Versorgung, die so komplex ist wie die psychosoziale Versorgung, kann deshalb nur aus der Populationsperspektive bewertet werden, etwa im Vergleich verschiedener Populationen in verschiedenen Versorgungsregionen.

\section{Fragen an die Versorgungsforschung \\ $\nabla$}

Unter Berücksichtigung dieser Besonderheiten der psychosozialen Therapien stellen sich folgende Forschungsfragen zur Umsetzung der neuen S3-Leitlinie:

\section{Welche psychosozialen Therapien werden von Klienten in Anspruch genommen?}

Psychosoziale Versorgung ist in der Gemeinde angelegt und funktioniert auch nur in der Kooperation regionaler Leistungserbringer wie Ärzten, Ergotherapeuten und psychosozialen Diensten. Die Untersuchungseinheit und die Einheit, in der Implementierung verglichen werden kann, muss daher die Region sein. Dabei spielen 3 Größen eine Rolle:

a) Die regionalen Versorgungsstrukturen: Welche Anbieter psychosozialer Hilfen sind in der Region vorhanden? Wie unterscheiden sich Regionen hinsichtlich der in ihnen vorhandenen psychosozialen Strukturen?

b) Die Prozesse: Inwieweit nehmen Klienten in einer definierten Region psychosoziale Therapien in Anspruch? Dies kann nur im Rahmen einer systematischen Befragung von Klienten erhoben werden. Dabei ist es weniger wichtig, von welchen Leistungserbringern die Klienten ihre Versorgung beziehen, sondern eher was sie tatsächlich an psychosozialen Versorgungsprozessen erhalten. Die in einer solchen versorgungsepidemiologischen Studie zu erfragenden Prozesse sollten sich an den in der Leitlinie empfohlenen Versorgungsprozessen orientieren.

c) Die Ergebnisse: Relevante Endpunkte und damit Parameter der Ergebnisqualität psychosozialer Versorgung umfassen unter anderem objektive Kriterien wie z.B. Heimeinweisung, Berentung, Aufnahme einer Arbeit, Krankenhauseinweisungen, sowie subjektive Kriterien wie gesundheitsbezogene Lebensqualität oder Recovery. Auch diese Ergebnisse sind nur aus Populationssicht und damit auf regionaler Ebene relevant.

\section{Was sind die Charakteristika von Klienten,} die psychosoziale Versorgung in Anspruch nehmen? Hier geht es vor allem darum, die Frage nach Unter-, Über- und Fehlversorgung zu beantworten. Dabei ist Überversorgung nicht nur unter dem Gesichtspunkt der Ressourcenallokation ein The- 
ma, sondern vor allem unter dem Aspekt von Abhängigkeit und Selbstständigkeit und Empowerment. Die Frage, wann eine ausreichende psychosoziale Versorgung erfolgt ist, wann und wie ein Ausstieg aus der Versorgung indiziert ist und wie dieser erfolgen soll, ist dabei zentral und in ihrer Brisanz nicht zu unterschätzen. Diese Fragen sind methodisch durch epidemiologische Untersuchungen der Charakteristika der Klienten anzugehen. Um die bisher wenig bearbeitete Frage nach der Beendigung von psychosozialer Versorgung und der Entlassung der Klienten in den eigenverantworteten Alltag anzugehen, sind hingegen eher qualitative Forschungsansätze geeignet.

\section{Welche Strukturen und Prozesse fördern eine} Implementierung der Leitlinie, wo sind Barrieren? Diese Frage kann durch Analysen und Vergleiche von Regionen, in denen es gut gelingt die Leitlinie zu implementieren mit Regionen in denen dies weniger gut gelingt angegangen werden. Dabei können je nach Datenlage Strukturen und Prozesse in den unterschiedlichen Regionen mit den klientenrelevanten Ergebnissen in Beziehung gesetzt werden. Eine andere methodische Möglichkeit, die etwas unabhängiger von der Qualität von Struktur-, Prozess- und Ergebnisdaten wäre, ist ein qualitativer Ansatz mittels Experteninterviews. Überall herrscht ein bestimmtes Wissen über Förderbedingungen und Barrieren zur Implementierung psychosozialer Versorgung vor. Allerdings ist dieses Expertenwissen immer eng verknüpft mit Eigeninteressen auf dem Markt der psychosozialen Therapien [6]. Die Herausforderung einer qualitativen Untersuchung dieser Frage wird daher eher in der Berücksichtigung der Vielfalt der Positionen und im Reflektieren der Unterschiedlichkeiten der Meinungen sein. Aufschlussreich könnte auch eine detaillierte Untersuchung relevanter Einstellungen der Behandler sein: welche Einstellungen und Haltungen führen zu einer Verordnung von leitliniengerechten psychosozialen Therapien? Hier stehen etablierte verhaltenstheoretische Modelle zur Verfügung [7], die bisher aber vor allem in Bezug auf traditionelle medizinische Maßnahmen angewandt wurden [8].

\section{Fazit}

$\nabla$

Die Leitlinien zu psychosozialen Therapien bei schweren psychischen Störungen weisen auf ein Gebiet der psychiatrischen Versorgung hin, das trotz seiner enormen Relevanz für das Wohlergehen der Betroffenen bisher von der Versorgungsforschung kaum beachtet worden ist. Die vorgestellten Überlegungen und Ansätze sollen dazu beitragen, dies zu ändern. Sie zeigen, dass sich anhand der Leitlinien relevante und realisierbare Forschungsansätze entwickeln lassen, die eine Umsetzung der Leitlinie unterstützen. Notwendig ist jetzt eine gezielte Förderung dieser Forschung.

\section{Danksagung}

Der Beitrag wurde durch das Referat psychosoziale Versorgungsforschung der Deutschen Gesellschaft für Psychiatrie und Psychotherapie, Psychosomatik und Nervenheilkunde (DGPPN) eingeladen und unterstützt.

Die komplette S3-Leitlinie sowie weitere relevante Publikationen mit Gliederung und PowerPoint-Präsentationen zum Download finden Sie unter: http://www.dgppn.de/dgppn/struktur/refera te/versorgung0/s3-leitlinie-psychosoziale-therapien-bei-schwe ren-psychischen-erkrankungen.html

\section{Literatur}

1 Ungewitter C, Bottger D, El-Jurdi J et al. Struktur und Kooperation in der Versorgung psychisch Kranker. Nervenarzt 2013; 84: 307-314

2 Amering M. Recovery ist eine Illusion - Pro \& Kontra. Psychiat Prax 2013; 40: 246-247

3 DGPPN. S3-Leitlinie Psychosoziale Therapien bei schweren psychischen Erkrankungen. Heidelberg: Springer; 2013

4 Becker T, Riedel-Heller SG. Neue alte Herausforderungen. Psychiat Prax 2012; 39: 365-366

5 Stengler K, Brieger P, Weig W. Psychiatrische Rehabilitation: „deutscher Sonderweg“ - wo geht es hin? Psychiat Prax 2010; 37: 206

6 Lucht ME, Schomerus G. E-Health in der Psychiatrie. Psychiat Prax 2013; 40: $301-303$

7 Ajzen I. The Theory of Planned Behavior. Organ Behav Hum Decis Process 1991; 50: 179-211

8 Schomerus G, Matschinger H, Angermeyer MC. Attitudes that determine willingness to seek psychiatric help for depression: a representative population survey applying the Theory of Planned Behaviour. Psychol Med 2009; 39: 1855-1865 